\title{
Measurement of Two Phase Flow
}

\begin{abstract}
J. Novotný, J. Nožička, J. Adamec, L. Nováková
This paper presents the results of experiments with moist wet steam. The aim of the experiment was to measure the velocity of the growth of a condensing nucleus in wet steam dependent on the velocity of condensation. For the experiments in wet steam an experimental setup was designed and constructed, which generated superheated steam at lowered pressure and a temperature of $50{ }^{\circ} \mathrm{C}$. Low pressure and temperature of the hot vapour was chosen in order to minimize the risk of accidental disruption of the wall. The size of the condensing nucleus was measured by the method of Interferometric Particle Imaging (IPI). The IPI method is a technique for determining the particle size of transparent and spherical particles based on calculating the fringes captured on a CCD array. The number of fringes depends on the particle size and on the optical configuration. The experimental setup used is identical with the setup for measuring flow by the stereo PIV method. The only difference is the use of a special camera mount comprising a transparent mirror and enabling both cameras to be focused to one point. We present the results of the development of the growth of a condensing nucleus and histograms of the sizes of all measured particles depending on position and condensation velocity.
\end{abstract}

Keywords: two phase flow, condensation, wet steam, IPI measurement.

\section{Introduction}

Since 2001, a PIV laboratory has been built up at the Department of Fluid Mechanics. The PIV system is now equipped with hardware and software for 3D measurements in air and water, concentration and temperature measurement in water, using the PLIF method, and two-phase flow measurements with the LIF and IPI methods. One of our partial goals is to verify the suitability of the IPI method for measuring of the size of the condensing cores in wet steam. We will project a simple course, build it and check its properties.

\section{Proposition of the measurement course}

For the desired outputs, we had to build a course line with the following properties:

- It has to be able to regulate the generated steam and its condensation in the measurement area

- The course has to be safe for the personnel

- The price has to be under $\$ 1000$

- It must be suitable for IPI measurement

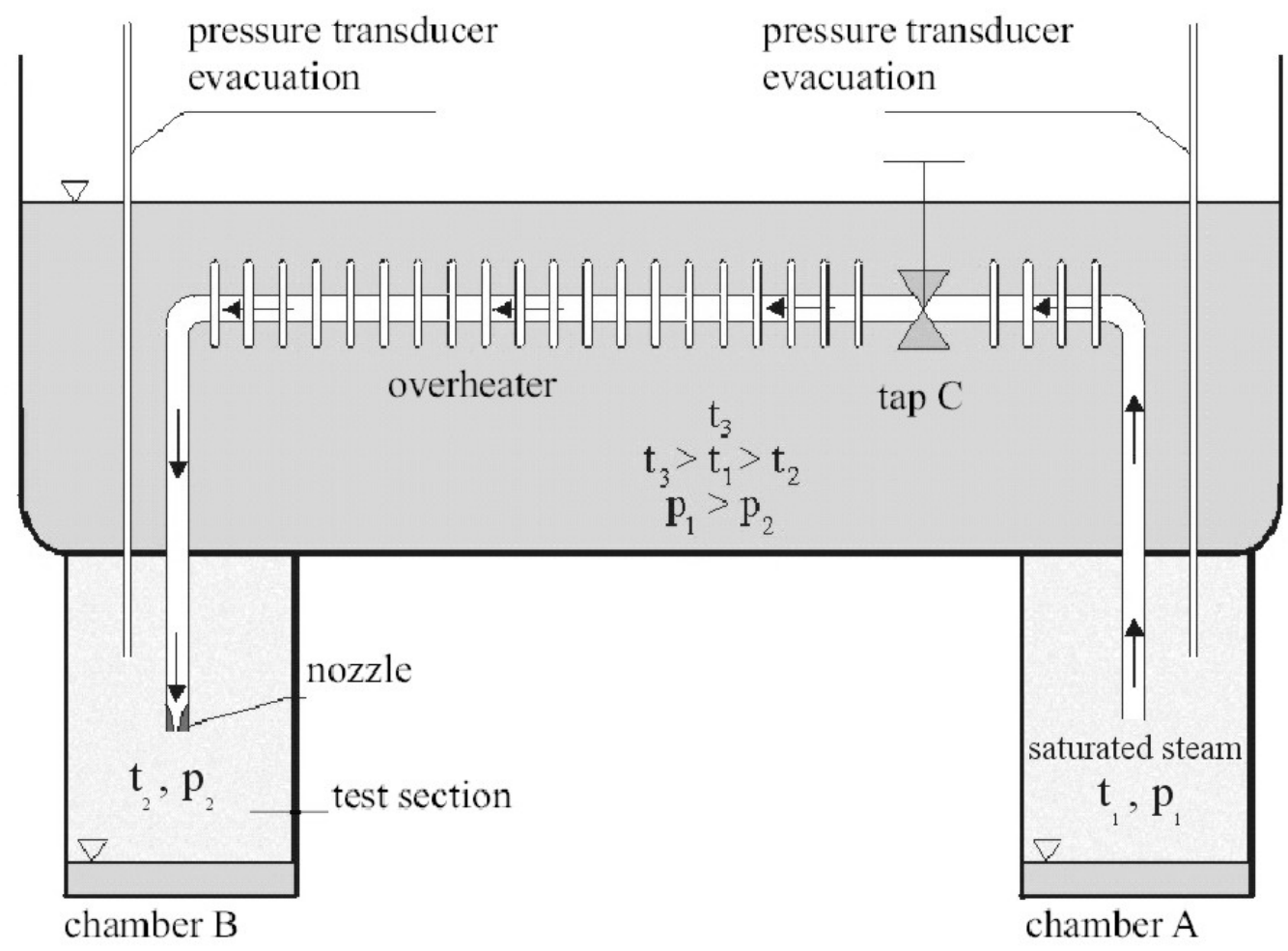

Fig. 1: Scheme of the measurement course 
For safety reasons we proposed a measurement course for low temperature superheated steam with lowered pressure. If the wall is damaged, there will be an implosion, which is much more acceptable than the possible damage from a regular explosion of a course filled with overheated steam at high pressure. The measurement course (Fig. 1) consists of two reservoirs (A and $\mathrm{B}$ ), which are connected with an overheater (cooler). Both chambers are filled to $1 / 10$ of their volume with water and drained to boiling point. The water in the Chamber A is warmer and so it needs higher pressure that in Chamber B to reach boiling point. Above the water surfaces there is in both cases saturated steam. Because of the higher pressure in Chamber A than in Chamber B, we get a sufficient hydraulic gradient for flow between the steam source and the measurement area. The pipe that connects the chambers has a jet with a 2-millimeter diameter. The two chambers chambers are separated with a tap (C). After exhaustion of both chambers and fixation of both pressures, stopcock $\mathrm{C}$ is opened and the saturated steam flows through the overheater into the measurement area with lower pressure steam. Here the steam condenses. Going though the overheater, the steam becomes a little hotter and overheated steam emerges. With the degree of overheating we regulate the beginning of condensation in the measurement area. With IPI the method and our laser (wave length $532 \mathrm{\eta m}$ ), we are able to measure only particles larger than $5 \mu \mathrm{m}$, and so the regulation at the beginning of the condensations essential, because the condensation cores are superfine at the beginning of the condensation. For the construction of the course we used a molten vessel with a volume of cca 31 as a source of warm steam. Measurement area with volume of a $20 \mathrm{l}$ was made of glass sheets $(10 \mathrm{~mm}$ in thicknees) and stiffened with crossbars. The vessel of the overheater (cooler) was made of brass sheet. Inside the overheater there was a ribbed pipe, which connects the chamber with "hot" steam and the measurement chamber. The pipe has a regulation tap, so that we are able to separate the chambers. The ribbed pipe is surrounded with water at acertain temperature, which has the function of a heat transfer medium. Both chambers have thermocouples and pressure pickups. The pressure and temperature of the water in the overheater can be set at will.

\section{Measurement method}

Interferometric Particle Imaging (IPI) is a measurement method based on getting the distance by measuring the interference fringes spaces on a picture of a defocused particle. We get the interference fringes in a picture of a defocused particle by illuminating the ball particle with a source of monochromatic light. Now there are to two reflections of the ray. On the front side the ray reflects light on the wall of the particle, and on the distant wall it first passes through the wall and then it reflects on the interface between the particle and the surrounding medium. The intensity of these two rays is the same, if we place the CCD camera at the right angle to the laser sheet. These two rays can be caught on the CCD chip as two light points. Because both light rays are generated at the same source and their routes were different at the moment of impact on the CCD chip, they are shift phased. If we defocus the lens of the CCD camera, both points will begin to generate characteristic Jang stripes. In the place where these stripes cross, we can see characteristic interference stripes. Their distance together with the optical setup of the lens and other parameters gives us the size of the particle. The principle for measuring the size of the particles is shown in Fig. 2.

We can obtain the particle diameter $d_{\mathrm{p}}=N_{\mathrm{fr}} / \kappa$ trough the following equation:

$$
\kappa=\frac{\arcsin \left(\frac{d_{a}}{2 z}\right)}{\lambda}\left(\cos \left(\frac{\phi}{2}\right)+\frac{m \cdot \sin \left(\frac{\phi}{2}\right)}{m^{2}+1-2 m \cdot \cos \left(\frac{\phi}{2}\right)}\right) .
$$

Where:

$$
\begin{array}{ll}
d_{\mathrm{p}} & \text { particle diameter, } \\
N_{\mathrm{fr}} & \text { number of fringes, } \\
\kappa & \text { geometric factor, } \\
d_{\mathrm{a}} & \text { aperture diameter, } \\
z & \text { distance from light sheet to camera lens, } \\
\phi & \text { observation angle, } \\
m & \text { relative index of refraction. }
\end{array}
$$

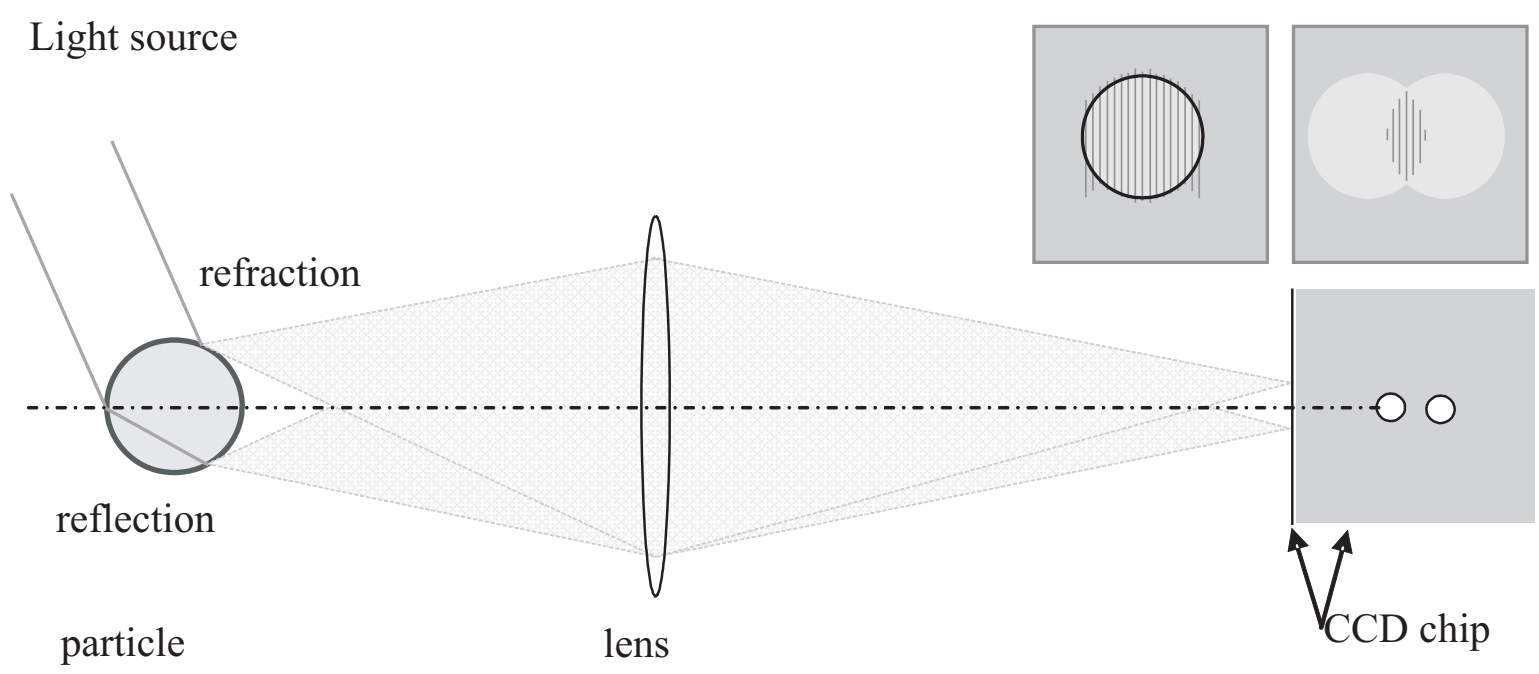

Fig. 2: The principle for measuring of the transparent and spherical particle. The pattern of the defocused particle with an interference fringe is shown in the right hand corner. 


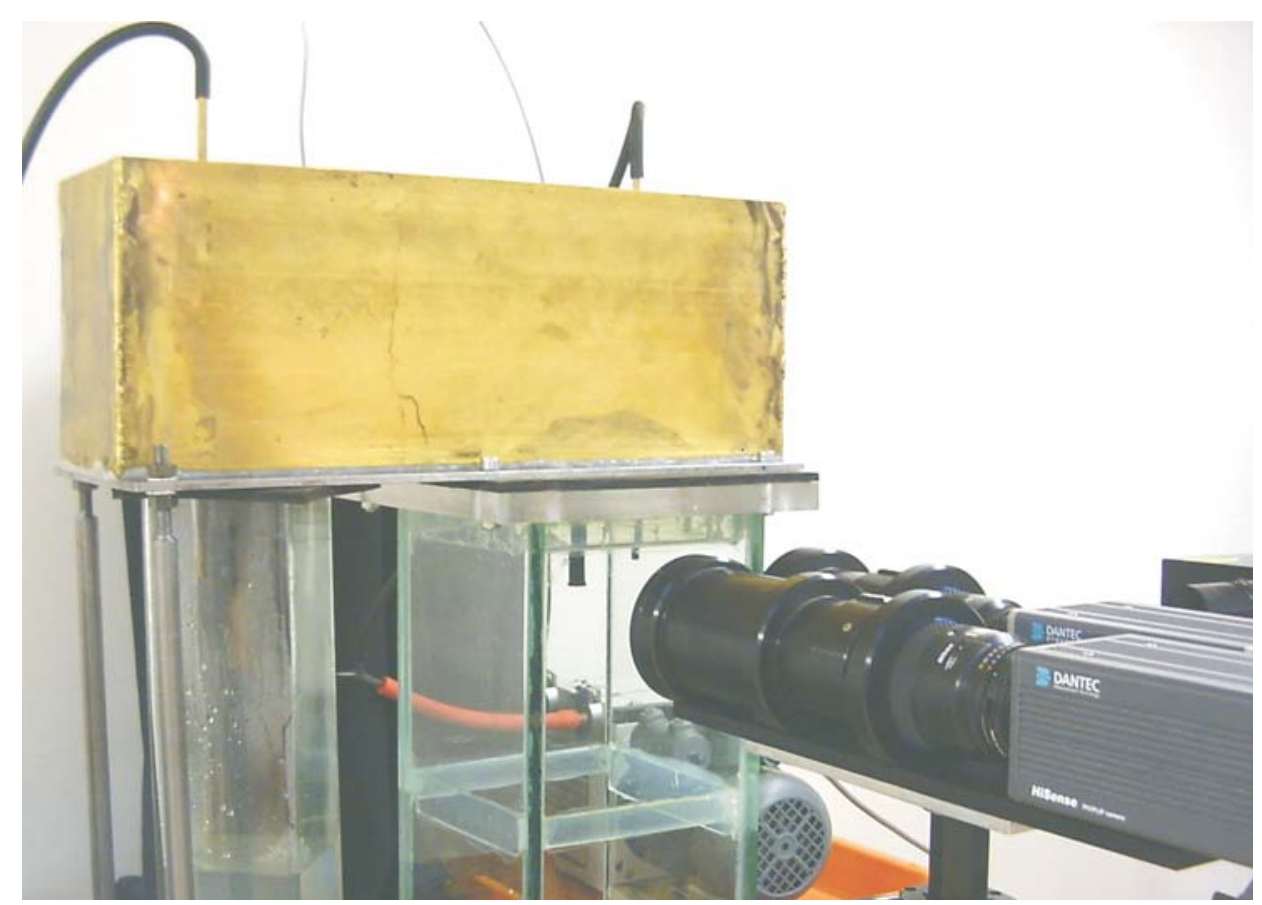

Fig. 3: Experimental adjustment
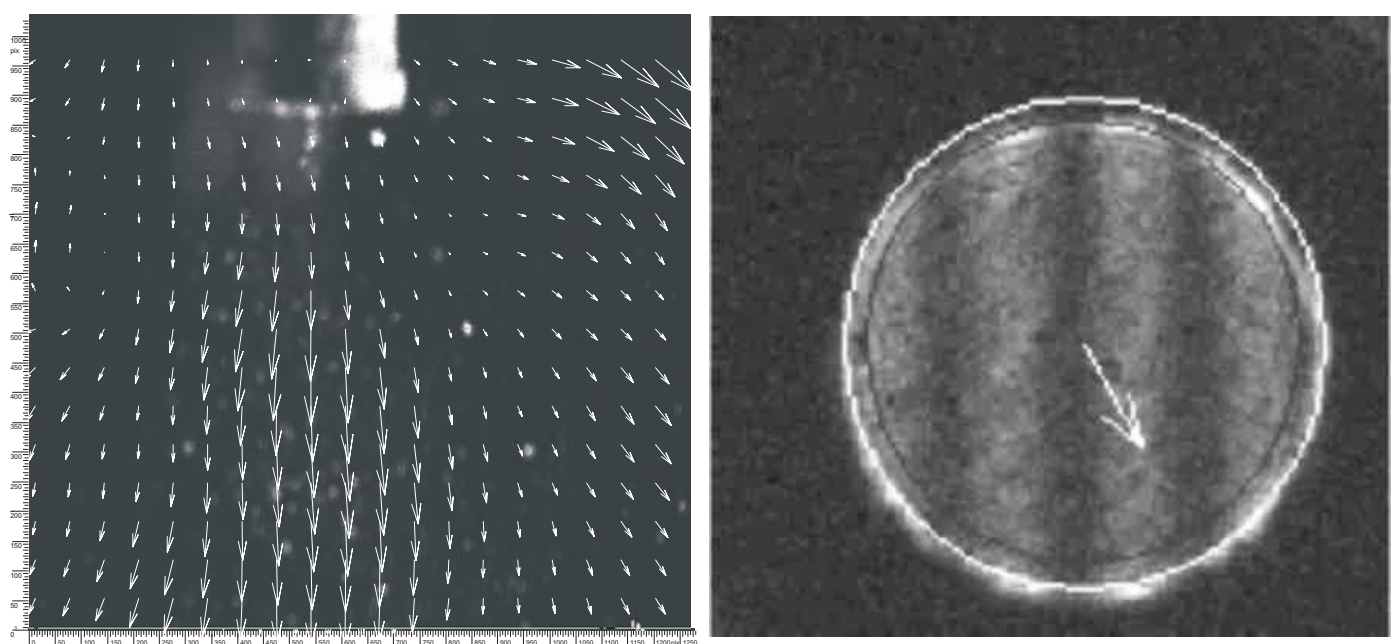

Fig. 4: Result of experiments. Instantaneous flow field and pattern of a defocused particle with an interference fringe. Detected position (outer white circle), diameter (inner gray circle), and velocity (arrow).

\section{Measurement}

The measured area delineated in the picture was $50 \mathrm{~mm}$ by $50 \mathrm{~mm}$ the size. Before the measurement itself, it was necessary to calibrate it with the help of a calibration chart placed in the measurement area. After successful calibration we performed several experiments with different setups of the temperatures: saturated steam in the measurement area and the source of "warm" saturated steam and even a different temperature in the overheater. Because of our $5 \mu \mathrm{m}$ limits in the size of the particle, we were not able to measure the size of the condensation particles using this setup. That's why we proceeded to the controlled condensation and we did not warm up the steam going through the overheater, but we were cooling it down. With this approach is possible to measure the size and placement of the condensating droplets of water, the results of some experiments are shown in the Fig. 4.

\section{Conclusion}

The course that we designed and built course gives us the opportunity to measure the size of condensing water droplets with the IPI method. Using our current laser beam, we are not able to measure size of the particles smaller than $5 \mu \mathrm{m}$ or particles that were placed too close to each other. In order to eliminate these insufficiencies it is necessary to have a camera with better CCD. With this camera we will be able to measure in a closer steam of particles. There is also a need to use a light source whit better properties for measurements at the beginning of condensation. The wavelength of this light source 
should be $100 \eta \mathrm{m}$. Finally the data should be compared with a numerical approach.

\section{References}

[1] Rafael, M., Willert, C., Kompenhans J.: Particle Image Velocimetry, Practical Guide. Berlin Heidelberg: Springer-Verlag, 1998.

[2] Pereira, F., Gharib, M., Dabiri, D., Modarress, D.: "Instantaneous Whole Field Measerument of Velocity and size of Air Microbubbbles in Two-Phase Flows DDPIV." $10^{\text {th }}$ International Symposium on Application of Laser Techniques to Fluid Mechanics, 10-13 July 2000, p. 38.4 .

[3] Alabria, R., Massoli, P.: "Experimental Study of Droplets in Evaporatin Regime by 2D Scattering Analysis." $10^{\text {th }}$
International Symposium on Application of Laser Techniques to Fluid Mechanics, 10-13 July 2000, p. 100-104.

Ing. Jan Novotný

Prof. Ing. Jiřri Nožička, CSc.

e-mail: jiri.nozicka@fs.cvut.cz

Doc. Ing. Jozef Adamec, CSc.

Department of Fluid Dynamics and Thermodynamics Ing. Ludmila Nováková

Department of Process Engineering

Czech Technical University in Prague

Faculty of Mechanical Engineering

Technická 4

16607 Prague 6, Czech Republic 